\title{
An atypical presentation of Kikuchi-Fujimoto disease mimicking systemic lupus erythematosus: case report and literature review
}

\author{
Diane Belder-Preston $^{1 *}$, Catherine-Maude Pound ${ }^{1,2}$, Roman Jurencak ${ }^{1,3}$ \\ ${ }^{1}$ Department of Pediatrics, Children's Hospital of Eastern Ontario, Ottawa, Canada; \\ ${ }^{2}$ Division of Pediatric Medicine, Children's Hospital of Eastern Ontario, Ottawa, Canada; \\ ${ }^{3}$ Division of Rheumatology, Children's Hospital of Eastern Ontario, Ottawa, Canada. \\ Email: ${ }^{\text {dianebelder@gmail.com }}$
}

Received 1 October 2011; revised 5 November 2011; accepted 18 November 2011.

\begin{abstract}
Purpose: To report a case of atypical Kikuchi-Fujimoto disease (KFD) that illustrates several overlapping features with systemic lupus erythematosus (SLE). Methods: A case is reported followed by a review of the current literature. Case Report: A 16-yearold boy with an unusual manifestation of KikuchiFujimoto disease (KFD) is described. The patient presented with fever, weight loss and severe abdominal pain, due to extensive necrotizing retroperitoneal and mesenteric lymphadenopathy. During the course of his illness, he developed several symptoms suggestive of systemic lupus erythematosus (SLE): a pericardial effusion, cotton wool spots on the retina and antibodies against nuclear antigens (ANA), Smith (Sm) and ribonucleoprotein (RNP) antigens. However, no additional features of SLE were found. The patient subsequently fully recovered within two months, without initiation of immunosuppressive therapy. His autoantibodies became negative five months after initial presentation and he remains well at his 23 month follow up visit. Discussion: We hypothesize that the autoantibodies developed by our patient were secondary to self-antigen induced autoimmunity related to his extensive tissue necrosis. Despite initially having clinical features suggestive of SLE, our patient's full and spontaneous recovery strongly supports the diagnosis of KFD. This illustrates the need for careful diagnosis, in order to avoid unnecessary and potentially toxic treatment with immunosuppressive agents.
\end{abstract}

Keywords: Kikuchi-Fujimoto’s Disease; Retroperitoneal Lymphadenitis; Systemic Lupus Erythematosus (SLE); Antinuclear Antibodies (ANA)

\section{INTRODUCTION}

Kikuchi disease, also called Kikuchi-Fujimoto Disease (KFD) and Histiocytic Necrotizing Lymphadenopathy, is a rare, benign, self-limited disease of unknown etiology. KFD was first reported in 1972 by Kikuchi [1] as well as independently, by Fujimoto et al. [2]. Typical features of the disease include subacute regional lymphadenopathy predominantly involving cervical lymph nodes as well as fevers, often accompanied by leukopenia, high erythrocyte sedimentation rate (ESR), and anemia [3]. Excisional biopsy of the affected lymph node shows nonspecific histopathologic features of cortical and paracortical necrosis. No specific diagnostic imaging or laboratory tests are available and KFD remains a diagnosis of exclusion. Clinicians must rule out other causes of necrotizing lymphadenopathy, such as malignancy, systemic connective tissue disorders, and infectious lymphadenitis, before diagnosing KFD. These other diagnoses have different therapeutic and prognostic implications. This can result in costly and invasive investigations, potentially causing significant distress to the patient and family.

We describe the case of a young man who presented to a pediatric tertiary care centre with atypical features of Kikuchi-Fujimoto Disease.

\section{CASE REPORT}

A 16-year-old, previously healthy, Caucasian boy presented to a pediatric tertiary care centre with a threeweek history of intermittent abdominal pain, vomiting, anorexia and a seven-kilogram weight loss. He also had a two-week history of intermittent fevers, up to 40 degrees Celsius, associated with a faint diffuse erythematous rash involving the face and upper torso. Personal and family histories were noncontributory.

Physical examination on admission revealed a pale, 
thin boy, weighing $59.7 \mathrm{~kg}$. His vital signs were: heart rate 120 , blood pressure $121 / 81$, respiratory rate 16 and oxygen saturation $98 \%$ in room air. He was afebrile with a faint malar flush. His abdomen was non-acute but diffusely tender with no hepatosplenomegaly. The remainder of the examination was unremarkable. No peripheral lymphadenopathy was appreciated.

Laboratory investigations showed elevated inflammatory markers, mild non-hemolytic anemia, transaminitis and markedly elevated lactate dehydrogenase (LDH) (Table 1). Autoantibodies (anti-nuclear antibodies (ANA), anti-neutrophil cytoplasmic antibodies, rheumatoid factor and antiphospholipid antibodies) were all negative. Complement (C3, C4) and total immunoglobulin levels (IgG, IgA and IgM) were within normal limits.

A Computerized Tomography scan of his abdomen demonstrated significant lymphadenopathy in both the retroperitoneum and the root of the mesentery. Explorative laporotomy with lymph node biopsy showed necrotizing lymphadenitis with extensive tissue necrosis, without evidence of neutrophilic inflammation or presence of granulomas. In one section, a vessel with intramural chronic inflammation suspicious for vasculitis was observed. Special stains for infectious organisms and immunophenotyping for neoplastic cells yielded negative results.

Given the absence of a definitive diagnosis, further investigations were performed. These included bone marrow studies to rule out malignancy, upper and lower endoscopies with biopsies to rule out atypical inflammatory bowel disease and Whipple's disease, as well as an extensive infectious work up. Tuberculosis, EpsteinBarr virus, toxoplasmosis, parvovirus, cytomegalovirus, Streptococcus pneumonia, and fungal infections were excluded. Ophthalmologic examination uncovered mul- tiple retinal cotton wool spots. Cardiac echocardiography demonstrated a moderate-sized pericardial effusion with no hemodynamic compromise. Magnetic Resonance Angiography of the abdomen, as well as a Magnetic Resonance Imaging of the hip and shoulder girdles helped to exclude the diagnoses of vasculitis and myositis.

During his hospitalization, our patient received mainly supportive care after a brief three-day course of a third generation cephalosporin antibiotic pending negative cultures. The severity of his abdominal pain was such that he required morphine, gabapentin, ketorolac and clonidine. Because of poor appetite, food intolerance and ongoing weight loss, total parenteral nutrition was initiated.

Atypical Kikuchi-Fujimoto disease was suspected based on the presence of necrotizing non-granulomatous retroperitoneal lymphadenitis and the exclusion of other disorders. Intravenous immunoglobulin therapy was offered but declined by the patient's family. Steroid therapy was not offered as there was still a degree of uncertainty in terms of the diagnosis of KFD at that stage.

With supportive management only, the patient's clinical status improved. By day 30 of hospitalization, he was ambulating, tolerating increasing naso-gastric feeds and demonstrating consistent weight gain. At the family's request, the patient was transferred to another tertiary care centre for a second opinion.

At the second hospital, approximately one month after his initial biopsy, the patient underwent another laporotomy with lymph node biopsy. Extensive bland necrosis with numerous macrophages was reported. Histiocytes, lymphocytes and minor plasma cell populations were scattered throughout. Again, no neutrophilic infiltration

Table 1. Laboratory data.

\begin{tabular}{|c|c|c|c|c|}
\hline & Reference range & During admission & Eight weeks after admission & Five months after admission \\
\hline ESR $(\mathrm{mm} / \mathrm{Hr})$ & $0-10$ & 63 & 13 & 6 \\
\hline CRP (mg/L) & $<8.0$ & 15 & $<1$ & $<1$ \\
\hline Ferritin (ug/L) & $24-336$ & 1408 & 968 & \\
\hline WBC $\left(\times 10^{9} \mathrm{~L}\right)$ & $4.5-11$ & 10.6 & 4.85 & 4.5 \\
\hline $\mathrm{Hb}(\mathrm{g} / \mathrm{L})$ & $120-160$ & 90 & 129 & 153 \\
\hline Plt $\left(\times 10^{9} \mathrm{~L}\right)$ & $150-450$ & 382 & 393 & 229 \\
\hline AST (U/L) & $14-50$ & 265 & 19 & 26 \\
\hline $\operatorname{ALT}(\mathrm{U} / \mathrm{L})$ & $10-55$ & 143 & 17 & 20 \\
\hline LDH (U/L) & $300-700$ & 3016 & 399 & 468 \\
\hline
\end{tabular}

ESR, erythrocyte sedimentation rate; CRP, C reactive protein; WBC, White blood cell; Hb, Hemoglobin; Plt, Platelet; AST, Aspartate aminotransferase; ALT, Alanine transaminase; LDH, lactate dehydrogenase. 
or granuloma was seen. Absence of hematoxylin bodies was noted. No infectious etiology was identified. On repeat testing, some of the patient's autoantibodies and immunoserologies became positive (Table 2). Laboratory methods used to detect these autoantibodies were identical at both hospitals (ANA by indirect immunofluorescence using Hep-2 cell line as substrate, ENA by ELISA). Nonetheless, he continued to improve with supportive treatment only, and no immunosuppressive therapy was initiated. There was full resolution of his pericardial effusion, and disappearance of the cotton wool spots on his retina.

The patient was discharged home with a presumed diagnosis of KFD. He now remains completely asymptomatic 23 months after hospital discharge. His previously abnormal inflammatory markers and positive immunoserologies have completely normalized (Tables $\mathbf{1}$ and 2).

\section{DISCUSSION}

In this report, we present a case of atypical KFD with retroperitoneal lymph node involvement and several features initially suggestive of SLE.

Most of the literature on KFD comes from the adult population, with the exception of several case series and reports published in the pediatric literature [3-10]. Young adults are predominantly affected, with a reported mean age of 25 years, as shown by a meta-analysis of 181 worldwide publications looking at 244 patients [7]. Cases have however been reported in all age groups, ranging from 19 months to 75 years [11]. Two recent pediatric case series, one from Korea [6] and the other from Taiwan [5], reported a mean age of 11 and 12.8 years respectively. Historically, there has been a higher predilection for young women, although a recent com- prehensive review reported a ratio closer to $1: 1$ [11]. In the pediatric population, the ratio seems to be higher in boys with reported ratios ranging from 1.4 to 2.8:1 [5, 6].

KFD typically presents with cervical lymphadenopathy. Low-grade fevers, fatigue, skin rashes affecting the face and the upper body, nausea, vomiting, diarrhea and weight loss are also reported [7,9,11]. Cases of non-cervical lymphadenopathy have been described; generalized, axillary, iliac and retroperitoneal lymphadenopathy (reported in 0 to $3 \%$ of cases) [4,12,13]. Rimar et al. [14] retrospectively reviewed 19 cases of possible KFD from seven medical centers in Israel between 1980 and 2007. In their study, a much higher rate of retroperitoneal and generalized lymphadenopathy was reported as compared to the rate reported in previous Far East studies. They found that $21 \%$ and $26 \%$ of cases presented with retroperitoneal and generalized lymphadenopathy, respectively.

Our patient presented with severe necrotizing abdominal lymphadenopathy without other lymph node involvement. Moreover, during the course of his illness, he developed several features suggestive of SLE (retinal vasculitis with cotton wool spots, small pericardial effusion and positive ANA, anti-Sm and anti-RNP antibodies). There are several case reports discussing an association between KFD and SLE [7,15-18] and some postulating KFD as a self-limited SLE-like autoimmune condition [19].

Our patient developed an autoantibody profile highly suggestive of SLE. However, his autoantibodies were positive only transiently and normalized within four months, without the need for immunosuppressive therapy. We hypothesize that the extensive necrotizing lymphadenopathy led to a release of self-antigens which, in

Table 2. Autoantibody testing.

\begin{tabular}{cccc}
\hline & On admission & Five weeks into the admission & Four months after initial positive results \\
\hline ANA & Negative & Positive & Negative \\
ANA titer & - & $1: 160$ & Negative \\
Anti-double stranded DNA & - & Negative & Negative \\
ENA screen & Negative & Positive & Negative \\
Anti Smith & - & Positive & Negative \\
Anti RNP & - & Positive & Negative \\
Anti Scl 70 & - & Negative & Negative \\
Anti Ro & - & Negative & Negative \\
Anti La & - & Negative
\end{tabular}

ANA, Anti-nuclear antibodies; RNP, Anti-ribonucleoprotein antibodies; ENA, Extractable Nuclear Antigens. 
the hyperinflammatory state, enabled mounting of a pathologic immune response with production of autoantibodies. Upon removal of the antigenic stimulation, the pathologic production of autoantibodies ceased, leading to their disappearance from the circulation. This hypothesis is supported by the fact that, 23 months after initial presentation, the patient remains off any therapy and healthy.

Cotton wool spots, which were observed in our patient, often indicate a serious systemic disease. They represent acute, focal, inner retinal ischemia and may occur in any disease that compromises arteriolar circulation to the inner retinal layers which includes SLE and systemic vasculitides where ischemia is caused by thrombogenic effects from antigen-antibody complexes [20]. However, similar to our patient, isolated, self-limited cotton wool spots in patients with no identifiable systemic disease have also been reported [21].

The presence of pericardial effusion in our patient could indicate SLE as pericarditis is the most common cardiac manifestation of this disease. However, transient asymptomatic pericardial effusions are often seen in instances of significant systemic inflammation, especially in the presence of accompanying hypoalbuminemia [22]. Our patient did have hypoalbuminemia (data not shown). The pericardial effusion in our patient was asymptomatic with spontaneous resolution. Pericarditis accompanying KFD has not been previously described.

Histologically, SLE and KFD can be very challenging to differentiate and at times impossible. In KFD, histopathological findings include varying degrees of necrosis, histiocytic proliferation with activated $\mathrm{T}$ lymphocytes, small lymphocytes and plasma cells without granulomatous inflammation with absent neutrophils and eosinophils. Adjacent vessels may be thrombosed. In contrast to KFD, SLE lymphadenitis, demonstrates hematoxylin bodies (aggregates of degenerated nuclear debris) and Azzopardi phenomenon (degenerated nuclear material aggregated in the walls of blood vessels). In SLE, abundant plasma cells, prominent reactive follicular hyperplasia, sparse cytoxic $\mathrm{T}$ cells and capsular and pericapsular inflammation are seen $[23,24]$. In our patient's biopsy specimens, the presence of extensive necrosis without neutrophilic inflammation or granulomas and the absence of hematoxylin bodies support the diagnosis of KFD rather than SLE or systemic vasculitis.

In conclusion, this report discusses a case of atypical KFD and illustrates several overlapping features of KFD and SLE. Our patient presented with retroperitoneal lymphadenopathy, an unusual manifestation of KFD. He demonstrated a transient elevation of autoantibodies typically associated with SLE, as well as retinal vasculitis and pericardial effusion, not previously described in patients with KFD. This illustrates the need for careful differential diagnosis, as KFD is self-limited and, contrary to SLE, does not require long-term immunosuppressive therapy; rather, often only supportive therapy is needed.

\section{REFERENCES}

[1] Kikuchi, M. (1972) Lymphadenitis showing focal reticulum cell hyperplasia with nuclear debris and phagocytes. Acta Haematologica Japanese, 35, 379-380.

[2] Fujimoto, Y., Kozima, Y. and Yamaguchi, K. (1972) Cervical subacute necrotizing lymphadenitis. Naika, 30, 920927.

[3] Han HJ, Lim G-Y, Yeo D-M., et al. (2009) Kikuchi’s disease in children: Clinical manifestations and imaging features. Journal of Korean Medical Science, 24, 11051109. doi:10.3346/jkms.2009.24.6.1105

[4] Saini, A.P., Jamil, S. and Dulai, M. (2010) Kikuchi's disease causing persistent fever and iliac lymphadenopathy in an 8-year-old boy. Clinical Pediatrics (Philadelphia), 49, 601-604. doi:10.1177/0009922809350497

[5] Lin, H.C., Su, C.Y. and Huang, S.C. (2005) Kikuchi’s disease in Asian children. Pediatrics, 115, e92-e96.

[6] Lee, K.Y., Yeon, Y.H. and Lee, B.C. (2004) KikuchiFujimoto disease with prolonged fever in children. Pediatrics, 114, e752-e756. doi:10.1542/peds.2004-0485

[7] Kucukardali, Y., Solmazgul, E., Kunter, E., et al. (2007) Kikuchi-fujimoto disease: Analysis of 244 cases. Clinical Rheumatology, 26, 50-54. doi:10.1007/s10067-006-0230-5

[8] Wang, T.J., Yang, Y.H., Lin, Y.T. and Chiang, B.L. (2004) Kikuchi-Fujimoto disease in children: Clinical features and disease course. Journal of Microbiology, Immunology and Infection, 37, 219-224.

[9] Chuang, C.H., Yan, D.C., Chiu, C.H., et al. (2005) Clinical and laboratory manifestations of Kikuchi's disease in children and differences between patients with and without prolonged fever. Pediatric Infectious Disease Journal, 24, 551-554. doi:10.1097/01.inf.0000167246.24500.21

[10] Payne, J.H., Evans, M. and Gerrard, M.P. (2003) Kikuchi-Fujimoto disease: A rare but important cause of lymphadenopathy. Acta Paediatrica, 92, 261-264. doi:10.1111/j.1651-2227.2003.tb00539.X

[11] Bosch, X., Guilabert, A., Miquel, R. and Campo, E. (2004) Enigmatic Kikuchi-Fujimoto disease: A comprehensive review. American Journal of Clinical Pathology, 122, 141-152. doi:10.1309/YF081L4TKYWVYVPQ

[12] Rudniki, C., Kessler, E., Zarfati, M., et al. (1988) Kikuchi's necrotizing lymphadenitis: A cause of fever of unknown origin and splenomegaly. Acta Haematologica, 79, 99-102. doi:10.1159/000205730

[13] Kita, Y., Kikuchi, M., Nakae, T., et al. (1997) A case of Kikuchi's disease with abdominal manifestations. Surgery, 122, 962-963. doi:10.1016/S0039-6060(97)90338-X

[14] Rimar, D., Zisman, D., Schendler, Y., et al. (2010) Kikuchi-Fujimoto disease in israel-more than a pain in the neck. Seminars in Arthritis and Rheumatism, 39, 515-520. doi:10.1016/j.semarthrit.2009.03.003 
[15] Martinez-Vazquez, C., Hughes, G., Bordon, J., et al. (1997) Histiocytic necrotizing lymphadenitis, KikuchiFujimoto's disease, associated with systemic lupus erythemotosus. QJM: An International Journal of Medicine, 90, 531-533. doi:10.1093/qjmed/90.8.531

[16] Fernandez, S.H. (2000) Kikuchi's lymphadenitis (necrotizing lymphadenitis) and systemic lupus erythematosus: A case report. Malaysian Journal of Pathology, 22, 25-29.

[17] Goldblatt, F., Andrew, J., Russell, A. and Isenberg, D. (2008) Association of Kikuchi-Fujimoto's disease with SLE. Rheumatology (Oxford), 47, 553-554. doi:10.1093/rheumatology/ken008

[18] Tsaousis, G., Kolevris, N., Vaidakis, E., et al. (1999) Kikuchi's disease and systemic lupus erythematosus: Case report and review. European Journal of Internal Medicine, 10, 53-55. doi:10.1016/S0953-6205(99)00010-2

[19] Nieman, R.B. (1990) Kikuchi's disease. Lancet, 335, 1563. doi:10.1016/0140-6736(90)91382-K
[20] Arevalo, J.F., Lowder, C.Y. and Muci-Mendoza, R. (2002) Ocular manifestations of systemic lupus erythematosus. Current Opinion in Ophthalmology, 13, 404-410. doi:10.1097/00055735-200212000-00011

[21] Remky, A. and Arend, O. (2000) Single isolated cottonwool spots. Ophthalmologica, 214, 143-148. doi:10.1159/000027485

[22] Sugiura, T., Kumon, Y., Kataoka, H., et al. (2008) Asymptomatic pericardial effusion in patients with rheumatoid arthritis. Cardiology, 110, 87-91. doi:10.1159/000110485

[23] Hu, S., Kuo, T.T. and Hong, H.S. (2003) Lupus lymphadenitis simulating Kikuchi's lymphadenitis in patients with systemic lupus erythematosus: A clinicopathological analysis of six cases and review of the literature. Pathology International, 53, 221-226. doi:10.1046/j.1320-5463.2003.01458.x

[24] Hutchinson, C.B. and Wang, E. (2010) Kikuchi-Fujimoto disease. Archives of Pathology \& Laboratory Medicine, 134, 289-293.

\section{ABBREVIATIONS}

Kikuchi-Fujimoto disease (KFD),

Systemic lupus erythematosus (SLE),

Antinuclear antibodies (ANA),

lactate dehydrogenase (LDH), erythrocyte sedimentation rate (ESR), smith (Sm),

ribonucleoprotein (RNP),

$C$ reactive protein (CRP),

White blood cell (WBC),

Hemoglobin (Hb),

Platelet (Plt),

Aspartate aminotransferase (AST),

Alanine transaminase (ALT). 